\title{
CD44 alternative splice variants v6 and v10
}

\author{
Ahmed Aquib*, Ajay Kumar Yadav** \\ * Dr. Rajendra Prasad Central Agricultural University, Pusa, Samastipur \\ ** Dr. B. R. Ambedkar Center for Biomedical Research, University of Delhi, New Delhi \\ **Corresponding Author: Dr. Ajay K. Yadav, email ID: ajay9774@gmail.com
}

\begin{abstract}
CD44 glycoprotein is ubiqutously present and situated at the extracellular surface of cell. Its role as an adhesion recptor was modified depending upon the state of post addition of glycomoeties under varied microenvironment. Many alternative spliced variants of CD44 were expressed by human cells depending upon the attainment during mutifunctional signal disposition. The function of splice factors, and hnRNPA family (Heterogenous ribonucleoproteins A) were dicussed in context to CD44 alternative variants. Glioma cell cline U87 MG were used in this study to search more about the affect of hnRNPA1 and hnRNPA2/B1 knockdown in CD44 V6 and CD44V10 expression. We found in preliminary study that splice factor knock down reduces the alternative splice variants of CD44 such as V6 \& V10. Therefore, potential of this study to elaborate further the mechanism in vivo model, to validate the alternative spliced variant in context to interaction with extracellular microenvironment.
\end{abstract}

\section{Introduction:}

CD44 a multifunctional extracelularly placed adhesion receptor, refer to as P-Glycoprotein. CD44 keep on modulated under the exposure to differential microenvironment, guiding cell to maintain homeostasis. Additional several posttranslational modifications like glycosylation under the various microenvironment presence, can be understand clearly through single amino-acid changes in polypeptide sequences of CD44. Many amino acid placed over the CD44 has many important role in addition to number of alternative splice variants of CD44. To study more about related the numreous amino acid undergoing modification has many contribution in exracellular as well as intrecellular CD44 activity. Site-directed mutagenesis is one of them strategy, by introducing precise changes in a DNA sequence with the aid of oligo-primers, introducing changes in some nucleotides [1]. A single basepair change in a nucleotide sequence refers to substitution, which can be used to map out the specific amino acid base interaction either by deletion or creating truncated mutations, function of a particular region in the protein sequences can be elucidated. Ankyrin binding sites of CD44 were the first to be predicted by mutational studies [16]. Thereafter, studies led to the identification of amino acid sequences important for the binding of other ligands of CD44.

\subsection{Identification of hyaluronan binding regions}

Hyaluronan, a high molecular weight polysaccharide, is a natural ligand of CD44. Two hyaluronan binding regions were identified by using truncation and site-directed mutagenesis of CD44s. A region homologous to another hyaluronan binding site near the amino terminus was recognized as one of the 
regions, whereas the other was located near the transmembrane region outside link-domain [2]. Two serine residues (S325 and S327) of CD44 cytoplasmic domain were reported to be responsible for phosphorylation of CD44 in $\mathrm{T}$ cells through mutational study. Point mutation of any of these residues impaired hyaluronan binding. Moreover, these substitutions also led to ligand-induced receptor modulation and disruption of adhesion to smooth muscle cells [3]. Site-specific mutagenesis investigations into two cysteine residues (286cys and 295cys) of CD44, present within the transmembrane domain, was done by Liu et.al. The substitution of the 286cys caused a reduction in hyaluronan binding [4]. Mutant LB cells having a substitution at the hyaluronan binding site of CD44v4-10 showed decreased in-vitro rolling and in-vivo local tumor growth while lymph node invasion vanished, suggesting that in-vitro and in-vivo cell migration is enhanced by CD44-HA interactions [5].

\subsubsection{Aldentification of utilized GAG motifs}

HS (Heparan Sulphate) and CS (Chondroitin Sulphate) are other GAGs that have the potential to modify cellular functions by interacting with CD44. Through site-directed mutagenesis and modification of full-length CD44, various splice variants were created. Transfectants expressing CD44v3 failed to bind to hyaluronan-coated surfaces but had a higher tumor developmental rate on intravenous injections [6]. CD44v3 also contains a Ser-GlySer-Gly motif that is the assembly point of GAGs such as HS (Heparan Sulphate) and CS (Chondroitin Sulphate). Serine to Alanine mutation of the Ser-Gly-Ser-Gly motifs eliminated HS and CS attachment, indicating that it is one of the utilized Ser-Gly-Ser-Gly motifs of CD44 [7]. An identical mutation of the Ser-Gly-Ser-Gly motif of CD44v3 was also demonstrated to be necessary for metastasis of
SKHep1 cells containing CD44v2-v10 [8]. An enhanced T-cell activation was achieved by generating artificial proteoglycan consisting of CD44 Ser-Gly-Ser-Gly motif fused with lymphocyte function-associated antigen-3 (LFA-3) and delivering it to human T-cells [8]. The artificial proteoglycan retained the natural function of LFA-3, engaging, and triggering CD2. Besides it also bound a chemokine RANTES (Regulated upon Activation, Normal T Cell Expressed and Presumably Secreted) [9].

\subsubsection{Identification of Amino Acids Functioning as c-Met co-receptor}

c-Met is a tyrosine kinase receptor that binds SC/HGF (Scatter Factor/ Hepatocyte Growth Factor) and influences invasive growth behavior and differentiation of cells. CD44v6 exon codes for 42 amino-acid long polypeptide chains. Three amino-acids in the center of CD44v6 were identified to be critical for its functioning as a co-receptor for c-met. Peptides having a mutation within these amino acids were unable to block c-Met activation and subsequent migration of cells [10]. Another study of CD44v6 was conducted by constructing a CD44v6 devoid of the cytoplasmic domain (CD44v6 $\Delta$ cyt). It was shown that in these cytoplasmic deletion mutants were unable to internalize c-Met [11]. HeLa cells transfected with CD44v6 mutant were tested for entry of beads coated with InIB, a protein required for entry of Listeria monocytogenes in mammalian cells. The mutant CD44v6 was able to obstruct the entry of InIB beads, indicating that InIB induced cmet activation also relies on CD44v6 [12].

\subsubsection{Identification of ERM Binding Amino Acid Clusters}

Interaction of CD44 to ERM (Ezrin, Radixin, and Merlin) proteins is another means through which CD44 influences cell signaling. Nine 
amino-acid long regions consisting of two positively charged amino acid clusters were identified within the CD44 cytoplasmic domain, to be the ezrin-binding site [13]. Sitedirected mutagenesis and immunoprecipitation confirmed the binding of ERM proteins to these clusters of positively charged amino acids near the transmembrane domain [14] ERM binding sites when removed from CD44 let to reduced hyaluronan binding in myeloid cells, suggesting that ERM proteins regulate hyaluronan binding in these cells [15].

\subsubsection{Recognition of Ankyrin Binding Site}

Ankyrin was the first identified intracellular protein having an interaction with CD44. Ankyrin binding and regulatory regions were identified through the analysis of mutant COS cells having deletions in the cytoplasmic domain of CD44. It was found that the region between amino acids 305 and 355 contains the ankyrin binding site [16]. A similar study reported that the deletion of 15 amino acid regions within the ankyrin binding site led to a decrease in hyaluronan mediated cell adhesion, Src kinase activity, and anchorage-independent growth of CD44-negative human prostate tumor cells (LNCaP). These findings suggested that ankyrin-CD44 interaction played a vital role in prostate tumor cell transformation [17].

\section{Impact of Alternative Splicing on CD44 pre-mRNA}

The majority of eukaryotic genes are alternatively spliced, providing the cell a great potential for protein diversity [18]. It is a critical mechanism for normal physiology as its dysregulation can have pathological consequences, including cancers [19]. CD44 protein is encoded by a single highly conserved gene present on chromosome 11. Its gene comprises 19 exons out of which 10 are consistent in all mature transcripts whereas others are alternatively spliced to give rise to a wide range of isoforms [20].

\subsection{Composition and Sequence Comparison of Alternatively Spliced Isoforms}

Alternative splicing in CD44 mainly affects the stem region of CD44 protein [21]. The standard isoform $(85-95 \mathrm{kDa})$ of CD44 consists of a 46 amino acid stem region, but the stem could be extended in transcript variants to up to 381 amino acids, as a result of the inclusion of variant exon product. The additional sequences can confer a further increase in molecular size (up to $250 \mathrm{kDa}$ ) by providing GAG attachment sites[22]. Variant exons (v1-v10), initially identified in the pancreatic rat cell line BSp73ASML, can be included singly or in different combinations to give rise to a multitude of transcript variants [23]. Theoretically, alternative splicing can generate 768 CD44 isoforms, but only up to 20 isoforms have been described [24]. The amino acid comparison revealed $64 \%$ homology of variant exons across species [25]. In addition to v1-v10 exons, the variation in CD44 molecule can also be attributed to the presence of internal splice sites in constant as well as variant exons. Among the constant exons are flanking exons $5 \mathrm{~s}$ and $6 \mathrm{~s}$ which contain internal splice sites [26]. Across species, the comparison revealed that flanking exons showed lesser sequence identity (54\% and $35 \%$ ) in comparison to the highly conserved identity $(82 \%-87 \%)$ of other constant exons. Variant exons $3 \mathrm{v}$ and $10 \mathrm{v}$ likewise contain additional splice sites and can, therefore, be present in variable lengths in mature transcripts. It's also worth noting that the variant exons (v1-v10) do not reveal any homology to another know protein [27].

\subsection{Occurrence and Distribution of CD44 Isoforms}

Both standard and variable isoforms of CD44 are expressed in a wide range of normal and malignant tissue [28]. The standard isoform is extensively expressed on a variety of tissue and 
is predominant. However, the larger variant isoforms appear on particular cell types of both normal and carcinomatous human tissues [29]. The longest variant of CD44 (CD44 v3-v10) was detected in keratinocytes. Whereas, CD44E (CD44 v8-10) or epithelial CD44 is expressed preferentially on epithelial cells [30]. CD44v6 exon containing isoforms occur in epithelial cells with extensive generating activities, such as glandular epithelium and basal cells of the stratified squamous epithelium [31]. Fetal generative and locomotive cells also express $\mathrm{CD} 44 \mathrm{v}$ isoforms, particularly isoforms containing v6 exon [32]. Similar isoforms also occur in malignant cells, which happen to share many properties to embryonic and adult proliferative cells. Overall CD44 expression is raised in activated and memory $\mathrm{T}$-cells, with a rise in isoforms containing $\mathrm{v} 9$ exon. Exon v6 is also reported to be upregulated during $\mathrm{T}$ cell activation and also after immunogenic activation of various other white blood cells [33].

\subsection{Functional Implication of CD44 Alternative Splicing}

Alternative splicing leads to the addition of single or multiple variant exons. Though there is only limited knowledge of CD44v exon product's structures, these additional motifs are thought to confer interactions with ligands in the microenvironment. Variant exons may impart additional features to isoforms, such as influencing HA binding, providing motifs for GAG binding, or binding or sequestering a growth factor. It was observed that if variant exon product is replaced by another protein sequence CD44-HA binding increases, even though CD44v contains a HA binding domain [34]. HA binding was also found associated with the amount of glycosylation due to alternative splicing [35].

The product of exon v3 contains a Heparan Sulphate binding site. The binding of HS to CD44 leads to its modification to Heparan Sulphate Proteoglycan (HSPG) [36]. Heparan binding growth factors, such as HBEGF (Heparan Binding Epidermal Growth Factor) and FGF (Fibroblast Growth Factor), attach to
HSPG and activate their receptors in turn [37], [38]. c-Met activation through SF/HGF (Scatter Factor/Hepatocyte Growth Factor) necessarily requires a CD44 variant isoform containing CD44v6. CD44v10 exon product contains the binding site for another GAG, chondroitin sulfate. Chondroitin sulfate attached CD44v10 is recognized by CD44 on other cells, thereby promoting cell-cell interaction in-vitro [39].

\subsection{Role of CD44 Alternative Splicing in Cancer Cells}

Splicing patterns of many genes including CD44 are altered as the cell moves through the oncogenic process. Although some tumors exclusively express the standard isoform of CD44, others may additionally or preferentially express a variant isoform. Isoform switching is thought to be a consequence of a response to some other stimuli in cancer as well as normal cells. Epithelial to mesenchymal transition (EMT) is an important step in the progression of cancer that confers stem-cell-like properties to tumor cells. The alternative splicing of CD44 is also regulated by EMT related factors [40]. Studies have shown the expression of variant isoforms in several types of cancers, and have found them to be associated with a poorer prognosis, drug resistance and survival of tumors [41], [42]. These ramifications are caused due to changes in signal transduction, ECM interactions, etc.

The CD44v isoforms have variable roles in tumor progression depending on the isoform and the type of tumor. CD44v6, an exon that promotes growth, was reported to play an important role in metastasis of colon and pancreatic cancers [43], [44]. CD44v3 was associated with migration, proliferation, and cisplatin resistance of head and neck squamous cell carcinoma [45]. Likewise, CD44v8-10 was found to be associated with lung colonization of breast cancer [46]. Gastric, prostate, and leukemia are among other cancers expressing variant isoforms of CD44 [47], [48], [49]. 


\section{Methodology}

\subsection{RBP Analysis}

RNA-binding proteins (RBP) determine the pattern of mRNA splicing. SpliceAid 2 is a database of human splicing factors expression data and RNA target motifs. This database was used to predict binding sites of splice factors on CD44 mRNA sequences. Three hundred nucleotide sequences from exon-intron junctions were entered in the RBP Map a computational tool to predict the RBP that bind to these sequences.[50] The sequences used were from the NCBI accession no. NM_000610.3. We selected the RBP involved in human splicing from the list of all RBP by matching the list with SpliceAid Database. The splice sites involved in splicing of Exon CD44v6 and CD44v10 were examined for the presence of binding sites of splice factors motifs. The graphical representation and the table of all the RBPs were reported.

\section{2 siRNA Transfection}

Glioma cells were transfected with siRNA against hnRNP A1 and hnRNP A2/B1 as per Santa Cruz Inc. protocol (Santa Cruz Inc.). Triton X-100 buffer was used to prepare lysates after 48 hours from transfection.

\subsubsection{Isolation of RNA from siRNA Treated Cell Lines}

U87MG are glioblastoma cell line used in brain cancer research. Total RNA from three siRNA treated U87 cells were used. TRIzol Reagent was used for extraction of total RNA, and the first-strand cDNA was generated.

\subsection{Primer Design and Synthesis}

Primers for qRT-PCR were designed using CD44 mRNA sequences from NCBI accession no. NM_000610.3. To design primers, the nucleotide sequence of CD44v6 and CD44v10 was uploaded in Primer-Blast [51]. From the primer blast results, the primer having suitable GC content and Tm was chosen. For efficient amplification in qRT-PCR cycles the amplicon length of $100 \mathrm{bp}$ to $200 \mathrm{bp}$ was chosen. The primer only showing predicted amplicons on intended target i.e. having high specificity was chosen. Table- 1 shows the primers used for qRT-PCR analysis and their respective amplicon size. All the primers used for qRT-PCR analysis were synthesized by IDT (Integrated DNA Technologies).

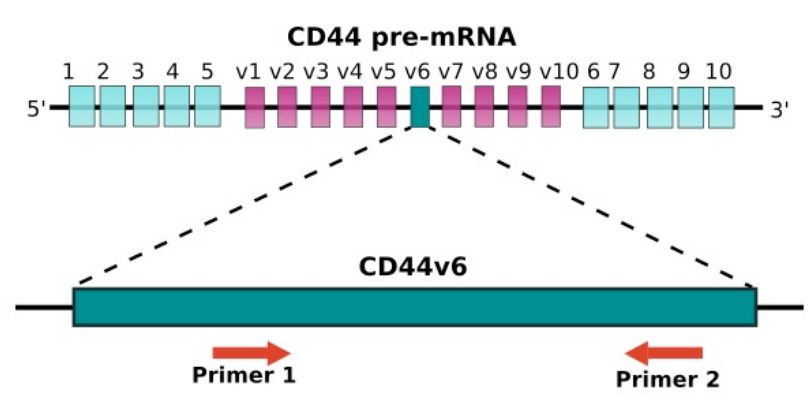

Figure 1: Primer pair position on CD44v6 exon.

Table -1: Primer sequences

\begin{tabular}{|l|l|l|c|l|}
\hline Primer & & $\begin{array}{l}\text { Sequences } \\
\mathbf{5}^{\prime} \text { to 3' }\end{array}$ & Length & Amplicon \\
\hline GAPDH & Forward & $\begin{array}{l}\text { AACGGGAA } \\
\text { GCTTGTCA } \\
\text { TCAATGGA } \\
\text { AA }\end{array}$ & 26 & 194bp \\
\cline { 2 - 4 } & Reverse & $\begin{array}{l}\text { GCATCAGC } \\
\text { AGAGGGGG } \\
\text { CAGAG }\end{array}$ & 21 & \\
\hline CD44v6 & Forward & $\begin{array}{l}\text { AACGGAAG } \\
\text { AAACAGCT } \\
\text { ACCA }\end{array}$ & 21 & \multirow{2}{*}{$100 \mathrm{bp}$} \\
\cline { 2 - 4 } & Reverse & $\begin{array}{l}\text { CCCTGTTG } \\
\text { TCGAATGG } \\
\text { GAGT }\end{array}$ & 20 & \\
\hline CD44v10 & Forward & $\begin{array}{l}\text { AGTGAAAG } \\
\text { GAGCAGCA } \\
\text { CTTCA }\end{array}$ & 21 & $131 \mathrm{bp}$ \\
\cline { 2 - 4 } & Reverse & $\begin{array}{l}\text { ACATCATTC } \\
\text { CTATTGGTA } \\
\text { GCAGGG }\end{array}$ & 24 & \\
\hline
\end{tabular}




\subsection{Quantitative Real-Time PCR}

Quantitative Real-Time PCR uses fluorescent reporters to determine absolute amount of a known sequence in a sample. In qRT-PCR, DNA amplification is monitored at each cycle of PCR. When the DNA is in the log linear phase of amplification, the amount of fluorescence increases above the background. A positive reaction is detected by accumulation of a fluorescent signal. The Ct (cycle threshold) is defined as the number of cycles required for the fluorescent signal to cross the threshold. EvaGreen $₫$ Supermix- Bio-Rad was used for SYBR Assay with cDNA and primers in 7300 thermocycler (Applied Biosystems). Glyceraldehyde-3-phosphate-dehydrogenase (GAPDH) gene served as an internal control. Thermal cycling consisted of a warm-up step of $2 \mathrm{~min}$ at $50^{\circ} \mathrm{C}$ and initial denaturation at $95^{\circ} \mathrm{C}$ for $10 \mathrm{~m}$, followed by 40 cycles of each polymerase chain reaction (PCR) step: $95^{\circ} \mathrm{C}$ for $15 \mathrm{~s}$ and $60^{\circ} \mathrm{C}$ for $1 \mathrm{~m}$.

\subsection{Statistical Analysis}

Experimental results have been expressed as the mean \pm standard error (S.E.). Statistical differences of data were assessed by Student's-test $p$ values lower than 0.05 were considered significant.

\section{Results}

An output summary of all predicted binding sites within one query sequence in a web-based presentation was retreived for both splice sites of exon CD44v6 and CD44v10. The results are provided for each of the proteins selected, where all the occurrences of motifs belonging to the same protein are listed together. A visualized presentation of the predicted binding sites as custom tracks in the UCSC Genome Browser is reported(Fig. 2). 


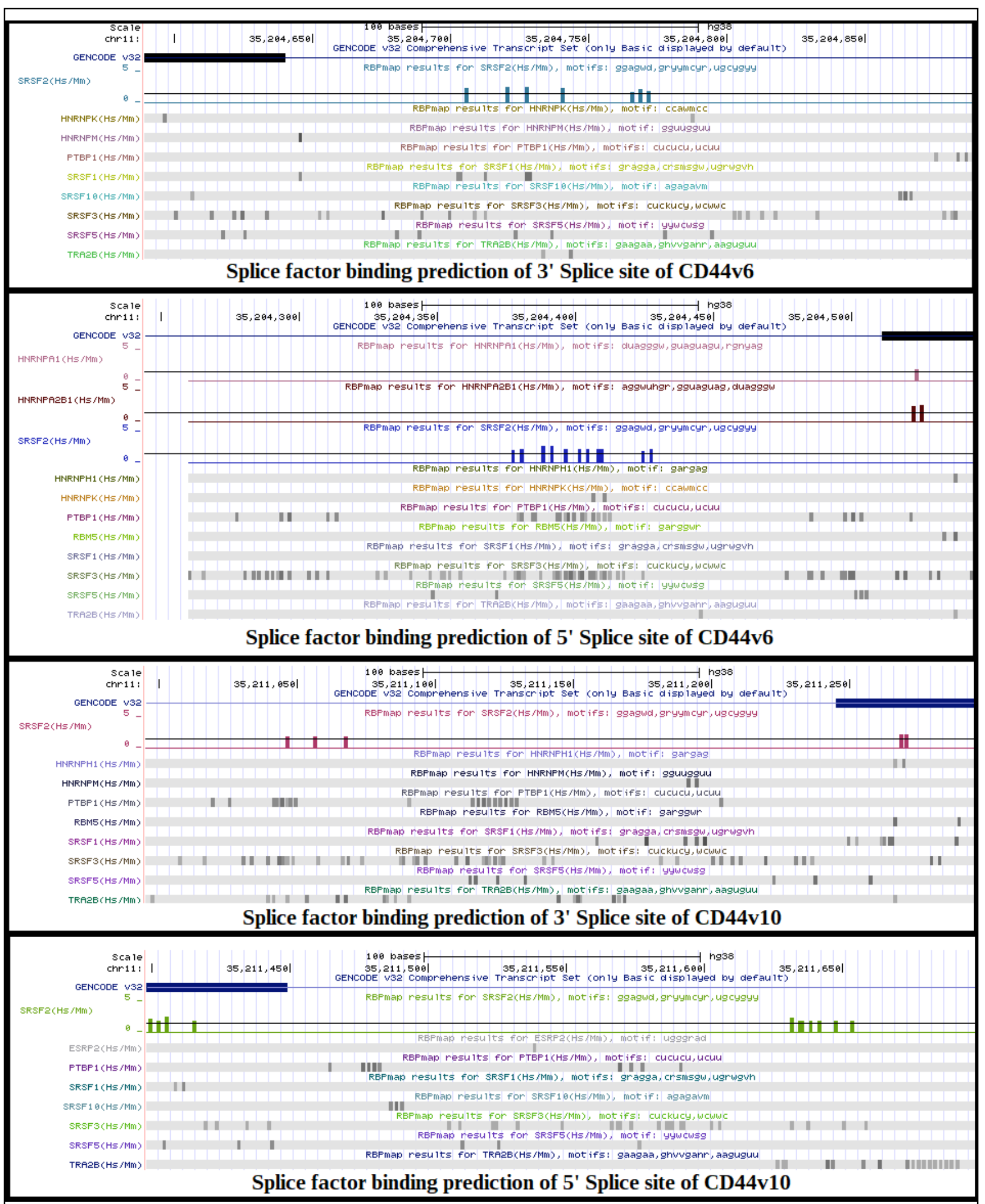

Figure 2: Splice Factor Binding Predictions for CD44v6 and CD44v10 Exons. 
In order to compare expression levels of CD44v6 in all the siRNA treated cells, the nfold values were calculated from cyclethreshold values (Fig-3). The expression of CD44v6 was the highest in non-knockdown (control) cells, while knock down of splice factors resulted in lower expression of exon v6. Similar results were obtained with CD44v10 exon (Fig-4), with non-knockdown cells having the highest expression.

\section{Conclusions and Discussions}

It was observed that knockdown of splice factors hnRNP A1 andhnRNP A2/B1 resulted in decrease in expression of CD44 variant exons in glioma cells. When these splice factors were not silenced the expression of CD44v6 and CD44v10 was highest. Therefore, these splice factors may be responsible for expression of these variant exons. Cancer cells display exceptional transcriptome alterations somewhat by utilizing cancer-specific splicing isoforms [52]. Mis-regulation of splicing is known to be a major contributor to various cancers. Many studies have confirmed that the misregulations of splicing could happen without mutation in target genes. Evidences suggest that these cancer-associated alternative splicing events occur due to change in splice factor expression [53].

The present study indicates more insights in splice factor dependent proliferation and metastasis, is also directed though alternative differential expression CD44 spliced varants. More therapeutic insights indeed require to understand the regulatory network upstream of overexpressed splice factor cancer tissues.

Conflict of Interest: No conflict of interest
Aknowledgement: Thanks Ms.Vidhi for sharing knock down RNA samples. Science and Engineering Research Board (SERB), New Delhi.

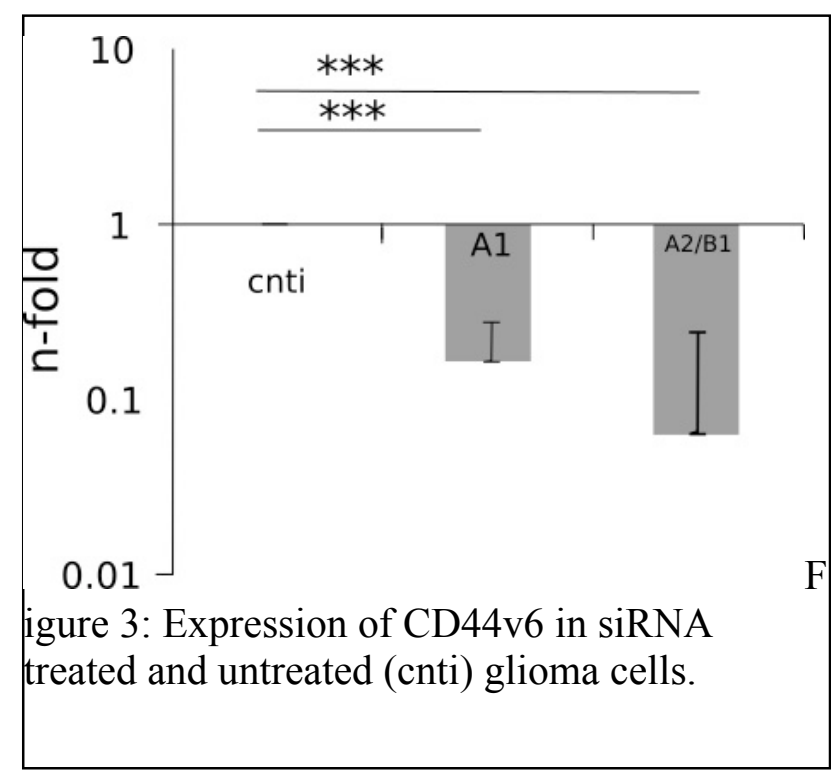




\section{REFERENCES}

[1] Ho, Steffan N., et al. "Site-directed mutagenesis by overlap extension using the polymerase chain reaction." Gene 77.1 (1989): 51-59.

[2] Peach, Robert J., et al. "Identification of hyaluronic acid binding sites in the extracellular domain of CD44." The Journal of cell biology 122.1 (1993): 257-264.

[3] Puré, Ellen, et al. "Defective phosphorylation and hyaluronate binding of CD44 with point mutations in the cytoplasmic domain." The Journal of experimental medicine 181.1 (1995): 55-62.

[4] Liu, Dacai, and Man-Syun Sy. "A cysteine residue located in the transmembrane domain of CD44 is important in binding of CD44 to hyaluronic acid." The Journal of experimental medicine 183.5 (1996): 1987-1994.

[5] Wallach-Dayan, Shulamit B., et al. "CD44-dependent lymphoma cell dissemination: a cell surface CD44 variant, rather than standard CD44, supports in vitro lymphoma cell rolling on hyaluronic acid substrate and its in vivo accumulation in the peripheral lymph nodes." Journal of cell science 114.19 (2001): 3463-3477.

[6] Bartolazzi, Armando, et al. "Regulation of growth and dissemination of a human lymphoma by CD44 splice variants." Journal of cell science 108.4 (1995): 1723-1733.

[7] Greenfield, Brad, et al. "Characterization of the heparan sulfate and chondroitin sulfate assembly sites in CD44." Journal of Biological Chemistry 274.4 (1999): 2511-2517.

[8] Barbour, Andrew P., et al. "Expression of the CD44v2-10 isoform confers a metastatic phenotype: importance of the heparan sulfate attachment site
CD44v3." Cancer research 63.4 (2003): 887-892.

[9] Wolff, Edith A., et al. "Generation of Artificial Proteoglycans Containing Glycosaminoglycan-modified CD44 DEMONSTRATION OF THE INTERACTION BETWEEN RANTES AND CHONDROITIN SULFATE." Journal of Biological Chemistry 274.4 (1999): 2518-2524.

[10] Matzke, Alexandra, et al. "A fiveamino-acid peptide blocks Met-and Ron-dependent cell migration." Cancer research 65.14 (2005): 6105-6110.

[11] Hasenauer, Susanne, et al. "Internalization of Met requires the coreceptor CD44v6 and its link to ERM proteins." PloS one 8.4 (2013).

[12] Jung, C., et al. "Involvement of CD44v6 in InlB dependent Listeria invasion." Molecular microbiology 72.5 (2009): 1196-1207.

[13] Legg, James W., and Clare M. Isacke. "Identification and functional analysis of the ezrin-binding site in the hyaluronan receptor, CD44." Current Biology 8.12 (1998): 705-708.

[14] Yonemura, Shigenobu, et al. "Ezrin/radixin/moesin (ERM) proteins bind to a positively charged amino acid cluster in the juxta-membrane cytoplasmic domain of CD44, CD43, and ICAM-2." The Journal of cell biology 140.4 (1998): 885-895.

[15] Brown, Kelly L., et al. "Regulation of hyaluronan binding by F-actin and colocalization of CD44 and phosphorylated ezrin/radixin/moesin (ERM) proteins in myeloid cells." Experimental cell research 303.2 (2005): 400-414.

[16] Lokeshwar, Vinata B., Nevis Fregien, and L. Y. Bourguignon. "Ankyrinbinding domain of CD44 (GP85) is required for the expression of hyaluronic acid-mediated adhesion 
function." The Journal of cell biology 126.4 (1994): 1099-1109.

[17] Zhu, Dan, and Lilly YW Bourguignon. "The ankyrin binding domain of $\mathrm{CD} 44 \mathrm{~s}$ is involved in regulating hyaluronic acid mediated functions and prostate tumor cell transformation." Cell motility and the cytoskeleton 39.3 (1998): 209-222.

[18] Fu, Xiang-Dong, and Manuel Ares Jr. "Context-dependent control of alternative splicing by RNA-binding proteins." Nature Reviews Genetics 15.10 (2014): 689-701.

[19] Chabot, Benoit, and Lulzim Shkreta. "Defective control of pre-messenger RNA splicing in human disease." Journal of Cell Biology 212.1 (2016):

13-27.

[20] Günthert, Ursula. "CD44: a multitude of isoforms with diverse functions." Adhesion in Leukocyte Homing and Differentiation. Springer, Berlin, Heidelberg, 1993. 47-63.

[21] Stamenkovic, Ivan, et al. "The hematopoietic and epithelial forms of CD44 are distinct polypeptides with different adhesion potentials for hyaluronate bearing cells." The EMBO journal 10.2 (1991): 343-348.

[22] Brown, Tod A., et al. "Human keratinocytes express a new CD44 core protein $(\mathrm{CD} 44 \mathrm{E})$ as a heparan-sulfate intrinsic membrane proteoglycan with additional exons." The Journal of cell biology $113.1 \quad$ (1991): 207-221.

[23] Screaton, Gavin R., et al. "Genomic structure of DNA encoding the lymphocyte homing receptor CD44 reveals at least 12 alternatively spliced exons." Proceedings of the National Academy of Sciences 89.24 (1992): 12160-12164.

[24] Van Weering, D. H., Pieter D. Baas, and Johannes L. Bos. "A PCR-based method for the analysis of human CD44 splice products." Genome Research 3.2 (1993):

100-106.

[25] Screaton, Gavin R., et al. "The identification of a new alternative exon with highly restricted tissue expression in transcripts encoding the mouse Pgp-1 (CD44) homing receptor. Comparison of all 10 variable exons between mouse, human, and rat." Journal of Biological Chemistry 268.17 (1993): 12235-12238.

[26] Shtivelman, E. M. M. A., and J. MICHAEL Bishop. "Expression of CD44 is repressed in neuroblastoma cells." Molecular and cellular biology 11.11 (1991): 5446-5453.

[27] Stamenkovic, Ivan, et al. "The hematopoietic and epithelial forms of CD44 are distinct polypeptides with different adhesion potentials for hyaluronate bearing cells." The EMBO journal 10.2 (1991): 343-348.

[28] Fox, Stephen B., et al. "Normal human tissues, in addition to some tumors, express multiple different CD44 isoforms." Cancer research 54.16 (1994): 4539-4546.

[29] Martegani, Marco Paolo, et al. "Structural variability of CD44v molecules and reliability of immunodetection of CD44 isoforms using mAbs specific for CD44 variant exon products." The American journal of pathology 154.1 (1999): 291-300.

[30] Günthert, Ursula, et al. "A new variant of glycoprotein CD44 confers metastatic potential to rat carcinoma cells." Cell 65.1 (1991): 1324.

[31] Stauder, Reinhard, and U. Günthert. "CD44 isoforms. Impact on lymphocyte activation and differentiation." Immunologist 3 (1995): 78-83.

[32]

Terpe, H-J., et al. "CD44 variant isoforms are preferentially expressed in basal epithelia of nonmalignant human fetal and adult 
tissues." Histochemistry 101.2 (1994): 79-89.

[33] Mackay, Charles R., et al. "Expression and modulation of CD44 variant isoforms in humans." The Journal of cell biology 124.1 (1994): 71-82.

[34]

Bennett, Kelly L., et al. "Regulation of CD44 binding to hyaluronan by glycosylation of variably spliced exons." The Journal of cell biology 131.6 (1995): 1623-1633.

Katoh, Shigeki, et al. "Glycosylation of CD44 negatively regulates its recognition of hyaluronan." The Journal of experimental medicine 182.2 (1995): 419-429.

[36]

Bennett, Kelly L., et al. "CD44 isoforms containing exon V3 are responsible for the presentation of heparin-binding growth factor." The Journal of cell biology 128.4 (1995): 687-698.

[37]

Sherman, Larry, et al. "A splice variant of CD44 expressed in the apical ectodermal ridge presents fibroblast growth factors to limb mesenchyme and is required for limb outgrowth." Genes \& development 12.7 (1998): 1058-1071.

$\mathrm{Yu}$, Wei-Hsuan, et al. "CD44 anchors the assembly of matrilysin/MMP-7 with heparin-binding epidermal growth factor precursor and ErbB4 and regulates female reproductive organ remodeling." Genes \& development 16.3 (2002): 307-323.

[39]

Cooper, David L., and

Graeme J. Dougherty. "To metastasize or not? Selection of CD44 splice sites." Nature medicine 1.7 (1995): 635637.

[40] Warzecha, Claude C., et al. "ESRP1 and ESRP2 are epithelial cell-type-specific regulators of FGFR2 splicing." Molecular cell 33.5 (2009): 591-601.

[41]

Ozawa, M., et al. "Prognostic significance of CD44 variant 2 upregulation in colorectal cancer." British journal of cancer 111.2 (2014): 365-374.

[42]

$\mathrm{Ni}$, Jie, et al. "CD44

variant 6 is associated with prostate cancer metastasis and

chemo /radioresistance." The

Prostate 74.6 (2014): 602-617.

[43]

Todaro, Matilde, et al.

"CD44v6 is a marker of constitutive and reprogrammed cancer stem cells driving colon cancer metastasis." Cell stem cell 14.3 (2014): 342-356.

[44] Rall, Christopher JN, and Anil K. Rustgi. "CD44 isoform expression in primary and metastatic pancreatic adenocarcinoma." Cancer Research 55.9 (1995): 1831-1835.

[45]

Wang, Steven J., Volkert

B. Wreesmann, and Lilly YW Bourguignon. "Association of CD44 V3 containing isoforms with tumor cell growth, migration, matrix metalloproteinase expression, and lymph node metastasis in head and neck cancer." Head \& Neck: Journal for the Sciences and Specialties of the Head and Neck 29.6 (2007): 550-558.

[46] Yae, Toshifumi, et al. "Alternative splicing of CD44 mRNA by ESRP1 enhances lung colonization of metastatic cancer cell." Nature communications 3.1 (2012): 1-9.

[47] Lau, Wen Min, et al. "CD44v8-10 is a cancer-specific marker for gastric cancer stem cells." Cancer research 74.9 (2014): 2630-2641.

[48]

Ghatak, Shibnath, et al. "Stromal hyaluronan interaction with epithelial CD44 variants promotes prostate cancer invasiveness by augmenting expression and function of hepatocyte growth factor and androgen receptor." Journal of Biological Chemistry 285.26 (2010): 19821-19832.

[49] Holm, Frida, et al. "Reversion to an embryonic alternative splicing program enhances leukemia stem cell self-renewal." Proceedings of the National Academy of Sciences 112.50 (2015): 15444-15449. 
[50] Paz I, Kosti I, Ares M Jr, Cline M, Mandel-Gutfreund Y. (2014) RBPmap: a web server for mapping binding sites of RNA-binding proteins. Nucleic Acids Res., 2014

[51] $\mathrm{Ye} J$, Coulouris G, Zaretskaya I, Cutcutache I, Rozen S, Madden T (2012). Primer-BLAST: A tool to design target-specific primers for polymerase chain reaction. BMC Bioinformatics. 13:134.
[52] El Marabti, Ettaib, and Ihab Younis. "The cancer spliceome: reprograming of alternative splicing in cancer." Frontiers in molecular biosciences 5 (2018): 80.

[53] Grosso, Ana Rita, Sandra Martins, and Maria Carmo Fonseca. "The emerging role of splicing factors in cancer." EMBO reports 9.11 (2008): 10871093. 